\title{
COVID-19 and flu: Dual threat, dual opportunity
}

$\mathrm{T}$ his fall, the Northern Hemisphere faces the dual threat of the annual influenza epidemic and the pandemic of coronavirus disease 2019 (COVID-19), which arrived here in March 2020 and never went away. But all is not bleak. Although the COVID-19 pandemic continues and has had a negative impact on preventive, routine, and acute medical care, the silver lining is that measures to combat the COVID-19 pandemic can help minimize the impact of seasonal influenza, and vice versa.

COVID-19 has justifiably occupied most of our attention in the last 10 months, but we need to keep our eyes on the ball of the upcoming flu season.

\section{COVID-19: THE ONGOING THREAT}

At the time of this writing, 1 million of the 33 million people with COVID-19 have died. ${ }^{1}$

The situation was similar in the 1918 influenza pandemic. Then, like now, no effective vaccine or medication was available to prevent or treat the pandemic disease. And despite significant advances in hygiene and supportive medical care achieved in the 100 years between these pandemics, the mortality rate during the early phase of the COVID-19 pandemic in New York City was comparable to that in the 1918 influenza pandemic. ${ }^{2}$

\section{Youth at risk}

During the early months of the COVID-19 pandemic, the incidence, disease severity, and mortality rates were highest among older adults. However, during June, July, and Au-

The author reports no relevant financial relationships which, in the context of his contribution, could be perceived as a potential conflict of interest. doi:10.3949/ccjm.87a.20161 gust 2020, the pattern shifted so that the incidence was highest in persons ages 20 to 29,3 and increases in incidence in this age group preceded increases in incidence in those age 60 and older by 4 to 15 days.

In the years 1999 to 2014, global surveillance data from 29 countries showed that young adults ages 18 to 39 accounted for $30 \%$ of influenza cases. ${ }^{4}$ Influenza vaccination coverage in the United States in adults ages 18 to 49 for the 2018-19 influenza season was 35\%, the lowest among all age groups. ${ }^{5}$ Even though disease severity of both COVID-19 and influenza may be less in healthy young adults than other age groups, it is becoming clear that the fight against both diseases may not be won without decreasing the incidence of both infections in that age group.

\section{Concurrent infection is common}

From March 3 to March 25 of this year, 20\% of specimens testing positive for severe acute respiratory syndrome coronavirus 2 (SARSCoV-2) were positive for 1 or more additional pathogens, including influenza. ${ }^{6}$ Numerous reports have since documented simultaneous SARS-CoV-2 and influenza co-infection. ${ }^{?}$

\section{COMPARING THE COVID-19} AND THE 2009 INFLUENZA PANDEMICS

The COVID-19 pandemic, the 2009 influenza A (H1N1) pandemic (the most recent flu pandemic), and seasonal influenza have several things in common that we need to recognize.

Table 1 summarizes similarities and differences between COVID-19 and 2009 influenza A (H1N1). ${ }^{8}$ Both have similar modes of transmission, but COVID-19 is much more contagious. Patients with COVID-19 are

\section{Measures to combat COVID-19 can help minimize the impact of seasonal influenza, and vice versa}




\section{TABLE 1}

\section{The coronavirus disease 2019 and influenza 2009 pandemics compared}

\begin{tabular}{|c|c|c|}
\hline & Coronavirus disease 2019 pandemic & Influ \\
\hline Causative virus & $\begin{array}{l}\text { Severe acute respiratory syndrome coronavirus } \\
2 \text { (SARS-CoV-2) }\end{array}$ & \\
\hline Mode of transmission & \multicolumn{2}{|l|}{$\begin{array}{l}\text { Respiratory droplets most common, but contact with } \\
\text { particles and airborne routes are possible }\end{array}$} \\
\hline Prevalence & $0.11 \%$ & $25 \%$ \\
\hline $\begin{array}{l}\text { Number of secondary transmis- } \\
\text { sions from } 1 \text { infected person }\end{array}$ & 2.5 & 1.7 \\
\hline Incubation period (days) & $2-14$ & 2 \\
\hline $\begin{array}{l}\text { Interval from symptom onset } \\
\text { to maximum infectivity (days) }\end{array}$ & 0 & 2 \\
\hline Duration of infectivity after & $8-10$ & $5-7$ \\
\hline
\end{tabular}

onset of illness (days)

Typical course of illness

Typical clinical presentation

Unique manifestations

Asymptomatic or presymptom-

atic

Illness requiring hospitalization

Illness requiring intensive care

Complications

Case fatality rate
Antiviral therapy
Dexamethasone therapy
Convalescent plasma therapy
Preventive measures other
than vaccine
Preventive vaccine

Pandemic duration
Gradual onset, then sudden escalation in severity, then recovery within 2 weeks in those with mild or moderate illness, and 3-6 weeks in those with severe illness
$25 \%$

1.7

$5-7$

Sudden onset of acute illness, which lasts $2-5$ days, followed by milder symptoms that can last for several weeks

Fever, headache, myalgia, malaise, and dry cough; less commonly, vomiting and diarrhea

Sudden loss of smell or taste None

$30 \%-40 \% \quad 20 \%$

$20 \% \quad 5 \%-10 \%$

$1 / 16,000 \quad 1 / 104,000$

Respiratory failure, myocarditis, encephalitis, myositis, multi-organ failure, and secondary bacterial pneumonia
$0.5 \%-1 \%$
$0.02 \%-0.05 \%$

Intravenous remdesivir (investigational)

Oral oseltamivir, inhaled zanamivir, intravenous peramivir, and oral baloxavir

Only if hypoxic

Not recommended

Investigational, only for severe cases

Social distancing, hand hygiene, face masks, isolation and contact tracing of confirmed cases, and quarantine of those exposed

In development; now 6 months into the Was developed and approved within 5 pandemic months

Ongoing; 8 months so far $\quad 15$ months

most contagious on the day of onset of symptoms, while those with 2009 pandemic influenza were most contagious during the first 2 days after the onset of illness. Asymptomatic or presymptomatic infections are up to twice as common with COVID-19 compared with influenza. Onset of illness with COVID-19 is usually gradual, while that of influenza is typically acute. Loss of sense of smell and taste are features of COVID-19, not influenza. Although the prevalence of influenza A (H1N1) during the 2009 pandemic was much higher than that of COVID-19 in the current pandemic, the latter is associated with up to 4 
times higher rates of hospitalization, 5 times higher rates of need for admission to an intensive care unit, and up to 10 times higher case-fatality, all possibly due to readily available, effective antiviral therapy for influenza.

\section{THE PANDEMIC'S NEGATIVE IMPACT ON OTHER MEDICAL CARE}

A major negative impact of social distancing measures to curb the COVID-19 pandemic is their indirect effect on preventive health care. Shortly after the pandemic was declared, rates of routine childhood immunizations in Michigan fell by about 20\%. ${ }^{9}$ Yet Abbas et a ${ }^{10}$ estimated that 84 deaths in African children would be prevented by sustaining routine childhood immunizations for every 1 excess COVID-19 death attributed to SARS-CoV-2 infections acquired during these routine vaccination clinic visits. The risk-benefit ratio extended to children's siblings, parents or adult caretakers, and older adults. This and other studies have led to a "call to action" to avoid the catastrophic negative impact the COVID-19 pandemic would have on vaccinepreventable diseases, including influenza. ${ }^{11}$

People are also avoiding routine and even urgent care. An estimated $40.9 \%$ of adults in the United States delayed or avoided routine medical care due to the COVID-19 pandemic. ${ }^{12}$ In the 10 weeks after declaration of a national emergency in the United States in response to the COVID-19 pandemic, emergency department visits for heart attack decreased by $23 \%$ and visits for stroke decreased by $20 \% .{ }^{13}$ Primary percutaneous coronary interventions for ST-segment elevation myocardial infarction decreased by $38 \%$ after March $1,2020 .{ }^{14}$

\section{MEASURES TO CONTAIN COVID-19 HELP CONTROL SEASONAL INFLUENZA}

Several teams around the world are at work on COVID-19 vaccines. Unfortunately, 31.6\% of adults surveyed indicated they were not sure they would accept such a vaccine, and $10.8 \%$ said they did not intend to be vaccinated. ${ }^{15}$ Not surprisingly, not having received the influenza vaccine the year before was one of the factors associated with vaccine hesitancy.

When a COVID-19 vaccine is approved, transparency and scientific integrity will be necessary to gain public trust and, hopefully, convince the hesitators and refusers. ${ }^{16} \mathrm{We}$ may need to choose our battles wisely by focusing on the slim majority $\left(57.6 \%{ }^{15}\right)$ who intend to be vaccinated, and by implementing measures well-established in annual influenza vaccination to close the intention-to-behavior gap, ${ }^{17}$ such as providing the vaccine free of charge at the workplace or school.

As an unexpected upside of the COVID-19 pandemic, the public health, nonpharmaceutical interventions for it such as social distancing, hand hygiene, face masks, isolation and contact tracing of confirmed cases, and quarantine of those exposed have resulted in collateral benefit on influenza activity. In the United States, within 2 weeks of the COVID-19 pandemic being declared on March 11, 2020, the percent of samples testing positive for influenza decreased sharply, from more than $20 \%$ before to $2.3 \% .^{18}$ In addition, interseasonal influenza circulation has remained at a historically low level of $0.2 \%$, compared with $1 \%$ to $2 \%$ in recent interseasonal periods. Moreover, data from several countries in the southern hemisphere ${ }^{18,19}$ and others such as Taiwan, ${ }^{20}$ Korea, ${ }^{21}$ Hong Kong, ${ }^{22}$ and Singapore ${ }^{23}$ indicate influenza activity in 2020 is at historically low levels.

Despite the negative psychological impact social distancing measures may have, recent surveys suggested that more Americans have embraced healthy lifestyles amid the COVID-19 pandemic. ${ }^{24,25}$

\section{MEASURES TO CONTROL SEASONAL INFLUENZA HELP FIGHT COVID-19}

Cross-reactivity of immune responses to influenza virus and coronavirus infections ${ }^{26}$ offers some insight into the potential beneficial effect of influenza vaccination on the COVID-19 pandemic. Similarity in structures and evolution of these viruses ${ }^{27}$ may explain this cross-reactivity of immunity. Another explanation is a "bystander immunity" induced by influenza vaccine against other viral infections. ${ }^{28,29}$

Preliminary data from more than 90,000 COVID-19 cases in Brazil showed that those who received influenza vaccine during the
A survey

in June 2020

showed that an estimated $40.9 \%$ of adults had delayed or avoided routine medical care due to the COVID-19 pandemic 
2020 influenza vaccination campaign, even after the onset of symptoms of COVID-19, were $8 \%$ less likely to require treatment in an intensive care unit, $18 \%$ less likely to require invasive respiratory support, and $17 \%$ less likely to die. ${ }^{30}$ The authors of this publication, which has not been peer-reviewed, cite adaptation in innate immunity as the most plausible mechanism for these beneficial effects.

Other intuitive beneficial effects of influenza vaccination during the COVID-19 pandemic include conserving resources, such as personal protective equipment, and more importantly healthcare providers. Healthcare systems were strained during the first several weeks of the pandemic, ${ }^{31}$ and all efforts to maintain this capacity should be implemented.

Variability in influenza vaccine efficacy ${ }^{32}$ has fueled continued suboptimal confidence in vaccination and therefore suboptimal vac- cination rates. ${ }^{33}$ Bartsch et al ${ }^{32}$ estimate that if we could produce a vaccine that was reliably $70 \%$ effective, it could avert up to 54 million influenza cases, saving up to $\$ 6.5$ billion in direct medical costs and up to $\$ 64.7$ billion in productivity losses. ${ }^{32}$

In an earlier article, ${ }^{34} \mathrm{I}$ addressed how to respond to influenza vaccine doubters. In addition, for those who believe there is an association between maternal vaccination during pregnancy and autism spectrum disorder in offspring, a recent article has refuted any such association. ${ }^{35}$

While patients with suspected or confirmed COVID-19 should postpone influenza immunization until they recover, ${ }^{36}$ everyone else 6 months of age and older who has no history of severe allergic reaction to any component if the vaccine, or to a previous dose of any influenza vaccine, should be immunized, preferably early in the flu season. ${ }^{37}$

\section{REFERENCES}

1. Worldometer. COVID-19 coronavirus pandemic. Accessed October 1, 2020. https://www.worldometers.info/coronavirus/

2. Faust JS, Lin Z, del Rio C. Comparison of estimated excess deaths in New York City during the COVID-19 and 1918 influenza pandemics. JAMA Netw Open 2020; 3(8):e2017527. doi:10.1001/jamanetworkopen.2020.1752

3. Boehmer TK, DeVies J, Caruso E, et al. Changing age distribution of the COVID-19 pandemic - United States, May-August 2020. MMWR Morb Mortal Wkly Rep 2020; 69(39):1404-1409.

doi:10.15585/mmwr.mm6939e1

4. Caini S, Spreeuwenberg P, Kusznierz GF, et al. Distribution of influenza virus types by age using case-based global surveillance data from twenty-nine countries, 1999-2014. BMC Infect Dis 2018; 18(1):269. doi:10.1186/s12879-018-3181-y.

5. Centers for Disease Control and Prevention. Flu vaccination coverage, United States, 2018-19 influenza season. FluVaxView webpage report posted online September 26, 2019. Accessed September 27th, 2020. https://www.cdc.gov/flu/fluvaxview/coverage-1819estimates. htm

6. Kim D, Quinn J, Pinsky B, Shah NH, Brown I. Rates of co-infection between SARS-CoV-2 and other respiratory pathogens. JAMA 2020; 323(20):2085-2086. doi:10.1001/jama.2020.6266

7. Cuadrado-Payán E, Montagud-Marrahi E, Torres-Elorza M, et al. SARS-CoV-2 and influenza virus co-infection. Lancet 2020; 395(10236):e84. doi:10.1016/S0140-6736(20)31052-7

8. Petersen E, Koopmans M, Go U, et al. Comparing SARS-CoV-2 with SARS-CoV and influenza pandemics. Lancet Infect Dis 2020; 20(9):e238-e244. doi:10.1016/S1473-3099(20)30484-9

9. Bramer CA, Kimmins LM, Swanson R, et al. Decline in child vaccination coverage during the COVID-19 Pandemic - Michigan Care Improvement Registry, May 2016-May 2020. MMWR Morb Mortal Wkly Rep 2020; 69(20):630-631. doi:10.15585/mmwr.mm6920e1

10. Abbas K, Procter SR, van Zandvoort K, et al. Routine childhood immunisation during the COVID-19 pandemic in Africa: a benefit-risk analysis of health benefits versus excess risk of SARS-CoV-2 infection. Lancet Glob Health 2020; 8(10):e1264-e1272. doi:10.1016/S2214-109X(20)30308-9

11. Hungerford D, Cunliffe NA. Coronavirus disease (COVID-19)
— impact on vaccine preventable diseases. Euro Surveill 2020; 25(18):2000756. doi:10.2807/1560-7917.ES.2020.25.18.2000756

12. Czeisler MÉ, Marynak K, Clarke KE, et al. Delay or avoidance of medical care because of COVID-19-related concerns - United States, June 2020. MMWR Morb Mortal Wkly Rep 2020; 69(36):12501257. doi:10.15585/mmwr.mm6936a4

13. Lange SJ, Ritchey MD, Goodman AB, et al. Potential indirect effects of the COVID-19 pandemic on use of emergency departments for acute life-threatening conditions - United States, January-May 2020. MMWR Morb Mortal Wkly Rep 2020; 69(25):795-800. doi:10.15585/mmwr.mm6925e2

14. Garcia S, Albaghdadi MS, Meraj PM, et al. Reduction in ST-segment elevation cardiac catheterization laboratory activations in the United States during COVID-19 pandemic. J Am Coll Cardiol 2020; 75(22) 2871-2872. doi:10.1016/j.jacc.2020.04.011

15. Fisher KA, Bloomstone SJ, Walder J, Crawford S, Fouayzi H, Mazor KM. Attitudes toward a potential SARS-CoV-2 vaccine: a survey of U.S. adults. Ann Intern Med Sep 4;M20-3569. doi:10.7326/M20-3569

16. Schwartz JL. Evaluating and deploying Covid-19 vaccines - the importance of transparency, scientific integrity, and public trust. N Engl J Med 2020 Sep 23. doi:10.1056/NEJMp2026393

17. Buttenheim AM. SARS-CoV-2 vaccine acceptance: we may need to choose our battles. Ann Intern Med 2020 Sep 4;M20-6206. doi:10.7326/M20-6206

18. Olsen SJ, Azziz-Baumgartner E, Budd AP, et al. Decreased influenza activity during the COVID-19 pandemic - United States, Australia, Chile, and South Africa, 2020. MMWR Morb Mortal Wkly Rep 2020; 69(37):1305-1309. doi:10.15585/mmwr.mm6937a6

19. Australian Government Department of Health. Australian influenza surveillance report and activity updates. Accessed October 1, 2020. https://www1.health.gov.au/internet/main/publishing.nsf/Content/ cda-surveil-ozflu-flucurr.htm

20. Kuo SC, Shih SM, Chien LH, Hsiung CA. Collateral benefit of COVID-19 control measures on influenza activity, Taiwan. Emerg Infect Dis 2020; 26(8):1928-1930. doi:10.3201/eid2608.201192

21. Lee $\mathbf{H}$, Lee $\mathbf{H}$, Song $\mathbf{K}-\mathbf{H}$, et al. Impact of public health interventions on seasonal influenza activity during the SARS-CoV-2 outbreak in Korea. Clin Infect Dis 2020;ciaa672. doi:10.1093/cid/ciaa672

22. Cowling BJ, Ali ST, Ng TWY, et al. Impact assessment of non-pharmaceutical interventions against coronavirus disease 2019 and in- 


\section{MOSSAD}

fluenza in Hong Kong: an observational study. Lancet Public Health 2020; 5(5):e279-288. doi:10.1016/S2468-2667(20)30090-6

23. Soo RJJ, Chiew CJ, Ma S, Pung R, Lee V. Decreased influenza incidence under COVID-19 control measures, Singapore. Emerg Infect Dis 2020; 26(8):1933-1935. doi:10.3201/eid2608.201229

24. Cleveland Clinic. Parade/Cleveland Clinic survey shows Americans embracing healthy lifestyle changes amid COVID-19 pandemic. Accessed October 9, 2020. https://newsroom.clevelandclinic. org/2020/09/25/parade-cleveland-clinic-survey-shows-americansembracing-healthy-lifestyle-changes-amid-covid-19-pandemic/

25. Pajer $\mathbf{N}, \mathbf{W e i n g u s} \mathbf{L}$. The results are in! Here are all the ways health and wellness look different in 2020. Parade 2020 Sep 25. https://parade.com/1088856/parade/cleveland-clinic-survey-results-2020/

26. Zheng J, Perlman S. Immune responses in influenza A virus and human coronavirus infections: an ongoing battle between the virus and host. Curr Opin Virol 2018; 28:42-52. doi:10.1016/j.coviro.2017.11.002

27. Zeng Q, Langereis MA, van Vliet ALW, Huizinga EG, de Groot RJ. Structure of coronavirus hemagglutinin-esterase offers insight into corona and influenza virus evolution. Proc Natl Acad Sci U S A 2008 105(26):9065-9069. doi:10.1073/pnas.0800502105

28. Horns F, Dekker LC, Quake SR. Memory B cell activation, broad anti-influenza antibodies, and bystander activation revealed by single-cell transcriptomics. Cell Rep 2020; 30(3):905-913. doi:10.1016/j.celrep.2019.12.063

29. Salem ML, El-Hennawy D. The possible beneficial adjuvant effect of influenza vaccine to minimize the severity of COVID-19. Med Hypotheses 2020; 140:109752. doi:10.1016/j.mehy.2020.109752

30. Fink G, Orlova-Fink N, Schindler T, et al. Inactivated trivalent influenza vaccine is associated with lower mortality among Covid-19 patients in Brazil. medRxiv 2020.06.29.20142505. doi:10.1101/2020.06.29.20142505

31. Blumenthal D, Fowler EJ, Abrams M, Collins SR. Covid-19 - implications for the health care system. N Engl J Med 2020 Jul 22. doi:10.1056/NEJMsb2021088

32. Bartsch SM, Mitgang EA, Geller G, et al. What if the influenza vaccine did not offer such variable protection? J Infect Dis 2020; 222(7):1138-1144. doi:10.1093/infdis/jiaa240

33. de Figueiredo A, Simas C, Karafillakis E, Paterson P, Larson HJ. Mapping global trends in vaccine confidence and investigating barriers to vaccine uptake: a large-scale retrospective temporal modelling study. Lancet 2020; 396(10255):898-908. doi:10.1016/S0140-6736(20)31558-0

34. Mossad SB. How to respond to flu vaccine doubters. Cleve Clin J Med 2019; 86(12):782-788. doi:10.3949/ccjm.86a.19139

35. Ludvigsson JF, Winell H, Sandin S, Cnattingius S, Stephansson O, Pasternak B. Maternal influenza A(H1N1) immunization during pregnancy and risk for autism spectrum disorder in offspring. Ann Intern Med 2020 Sep 1. doi:10.7326/M20-0167

36. Centers for Disease Control and Prevention. Vaccination guidance during a pandemic. Accessed October 9, 2020. https://www.cdc.gov/ vaccines/pandemic-guidance/index.html

37. Grohskopf LA, Alyanak E, Broder KR, et al. Prevention and control of seasonal influenza with vaccines: recommendations of the Advisory Committee on Immunization Practices - United States, 2020-21 influenza season. MMWR Recomm Rep 2020; 69(8):1-24. doi:10.15585/mmwr.rr6908a1

Address: Sherif Beniameen Mossad, MD, Department of Infectious Dis eases, G21, Cleveland Clinic, 9500 Euclid Avenue, Cleveland, OH 44195; mossads@ccf.org 